\title{
Substrate Deprivation Therapy
}

National Cancer Institute

\section{Source}

National Cancer Institute. Substrate Deprivation Therapy. NCI Thesaurus. Code C85876.

A therapeutic procedure based on the inhibition or reduction of the biosynthesis of compounds that cannot break down in the cells. 